\title{
Using polynomial modeling for calculation of quality parameters in computer assisted sperm analysis
}

\author{
Fatih OKUMUŞ ${ }^{*}$ iD , Adnan Fatih KOCAMAZ ${ }^{2}$ iD , Mustafa Erkan ÖZGÜR ${ }^{3}$ iD \\ ${ }^{1}$ Department of Software Engineering, Inonu University, Malatya, Turkey \\ ${ }^{2}$ Department of Computer Engineering, Inonu University, Malatya, Turkey \\ ${ }^{3}$ Department of Fisheries, Malatya Turgut Ozal University, Malatya, Turkey \\ (fatih.okumus@inonu.edu.tr, fatih.kocamaz@inonu.edu.tr, mustafa.ozgur@ozal.edu.tr)
}

$\begin{array}{lll}\text { Received: Sep.22, } 2021 & \text { Accepted: Oct.9, } 2021 & \text { Published: Dec. 1, } 2021\end{array}$

\begin{abstract}
Measurement of sperm velocity parameters takes an important place in sperm analysis. Today, computer-assisted sperm analysis (CASA) systems are used to detect motility measurements such as VCL (Curvilinear Velocity), VSL (Linear Velocity) and VAP (Average Path Velocity). The path length of sperm cells is necessary to calculate the motility parameters and it is calculated using video processing. However, this path length is obtained by the discrete-time processing of video frames, which can lead to unrealistic results. In CASA systems, the frame rate of the videos is increased to obtain the natural path length, but in this case, it is necessary to increase the hardware cost as well. Polynomial modelling applied to measure the natural path length in discrete-time structures can solve this problem. In this study, Lagrange interpolation which is an effective polynomial modelling method was used to obtain the natural path length and velocity parameters of the sperm. The results of the study showed that, when applied the polynomial modelling to calculate VCL and VAP parameters, it was found that especially in the low frame rate, more effective data were obtained than the classical method. As a result of this study, it is recommended to use polynomial modelling in the sperm velocity calculations of CASA systems, although there is an increase in calculation time.
\end{abstract}

Keywords : Tracking (time series analysis), Polynomial modeling, Sperm motility, Lagrange interpolation, Computer-assisted detection and diagnosis.

\section{Introduction}

It is known that sperm quality is the main cause of reproductive function diseases such as infertility in men (Qi et al., 2019). Sperm analysis methods are applied on frozen sperm samples to measure sperm quality (Contri et al., 2010). As a result of the analysis, concentration, motility ratios, velocity parameters and morphological characteristics of sperm cells are obtained. Velocity values of sperm cells are: VCL (Curvilinear Velocity), VSL (Linear Velocity), VAP (Average Path Velocity), ALH (Mean Amplitude of Lateral Head Displacement), BCF (Frequency of Head Displacement), LIN (Linearity Coefficient), STR (Straightness Coefficient), WOB (Wobble Coefficient), MAD (Mean Angular Displacement) (Amann and Waberski, 2014; Duffy et al., 2015; Hu et al., 2019; Kř́žková et al., 2017; Yániz et al., 2015). These parameters are also accepted by the World Health Organization (WHO) (World Health Organization, 2010). In Fig. 1, the VCL, VSL, VAP and ALH parameters are simulated. 


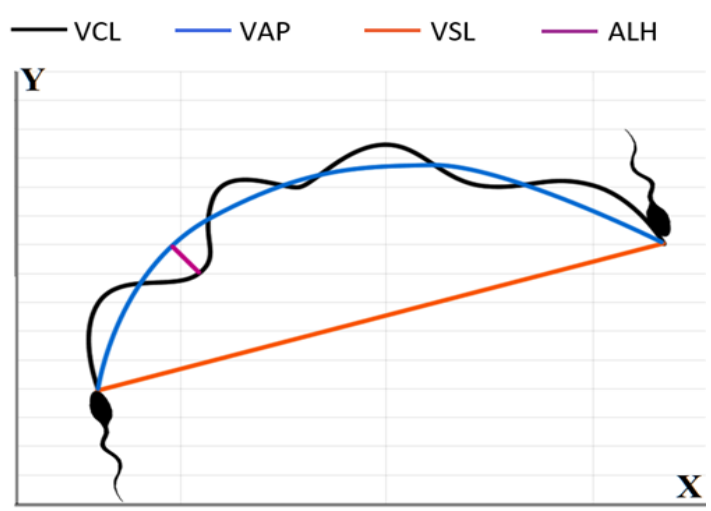

Figure 1. Some of Sperm Velocity Parameters (VCL, VAP, VSL, ALH)

Computer-Assisted Sperm Analysis (CASA) systems are one of the methods of measuring sperm quality and use computer vision methods (Boe-Hansen and Satake, 2019; Rurangwa et al., 2004). They are generally produced by commercial enterprises and offered as proprietary software. Since the late 1970s, many studies have been conducted in this field (Bompart et al., 2018). Wilson-Leedy and Ingermann provided an open source CASA system in their study (Amann and Waberski, 2014; WilsonLeedy and Ingermann, 2007). Özgür et al. proposed a computer-assisted sperm analysis system that measures sperm motility and speed parameters in fish and presented the algorithmic flow of their studies (ÖZGÜR et al., 2019). Urbano et al. presented a fully automated multi-sperm tracking algorithm (Urbano et al., 2017). Progress can also be seen in the CASA systems produced with the development of technology. Yamasaki et al. showed that a portable CASA system could be implemented using smartphones (Yamasaki et al., 2017). Alquézar-Baeta et al. have developed open source software called OpenCASA (Alquézar-Baeta et al., 2019). Khalifa et al. have performed a study that allows the researchers to process the video in real time and perform multi-tracking (Khalifa et al., 2019).

In CASA systems, VCL is obtained by combining all the points in the frames from the beginning to the end of the sperm video. The average path velocity, VAP is calculated by taking the average of VCL values (Amann and Waberski, 2014; Bompart et al., 2018; Hidayatullah et al., 2015; Nieschlag and Behre, 2001). These parameters are simulated in Fig.1. When calculating the VCL and VAP parameters, there is a curvilinear path, unlike other parameters. Unwanted results can be achieved when this curvilinear path is not considered in real time.

In CASA systems, video processing is performed to analyze the path and behavior of the sperm through the video. Since the video contains a lot of sperm, multiple objects tracking algorithms are used to classify all moving objects. Background and foreground extraction of pixels in all frames is required before video processing (Ahn and Cho, 2019; Elgammal et al., 2002; Stauffer and Grimson, 1999). Background subtraction classifies the pixels found in each frame of a video sequence. Background pixels belong to constant objects throughout the video, and the others belong to moving objects (Bouwmans, 2014; Cocorullo et al., 2016; Sobral and Vacavant, 2014). Multiple object tracking systems use machine learning methods to find out which pixels belong to which moving object. The CASA system takes the center point of the head region of the sperms detected by machine algorithms and shows the path in the (x, y) plane (ÖZGÜR et al., 2019).

The loss of time between the two frames, due to the FPS feature of the video, leads to a discretetime calculation of the sperm path. The discrete-time path formation will not give the real path for the sperm in the time interval between the two frames. There is an error percentage based on the number of transition points. However, a video with a high Frame per Second (FPS) will produce a near-realistic result because the interval between two frames will be shorter. Since the time passing between two frames in a video taken with a camera having a low FPS value will be long, the path taken by the sperm will be computed differently from the camera with a high FPS. This problem occurs in the process of obtaining kinematic and curvilinear parameters such as VAP and VCL. At the same time, the other characteristic values that vary according to the values of these two parameters are affected by this situation. Bompart et al. stated that the frame rate is one of the important issues in sperm analysis and it will be effective in the calculation of kinematic parameters such as VCL (Bompart et al., 2018). Wilson- 
Leedy and Ingermann noted that the VCL parameters generated by varied FPS records are different from each other (Wilson-Leedy and Ingermann, 2007). Valverde et al. investigated the optimal frame rate for some farm animal, suggesting that frame rates on videos might affect the quality of data obtained (Valverde et al., 2019).

In Fig.2.a, the real-time path of a sperm cell is simulated. As seen in the image, the path followed by the sperm cell in a continuous time system does not have sharp bends. However, CASA calculates the sperm path according to the point location information in each frame. In Fig.2.b, it can be seen that the path of a cell in different FPS records can be estimated with different lengths. It seems that the reliability of data obtained by measuring sperm velocity information depends on the FPS feature of the camera.

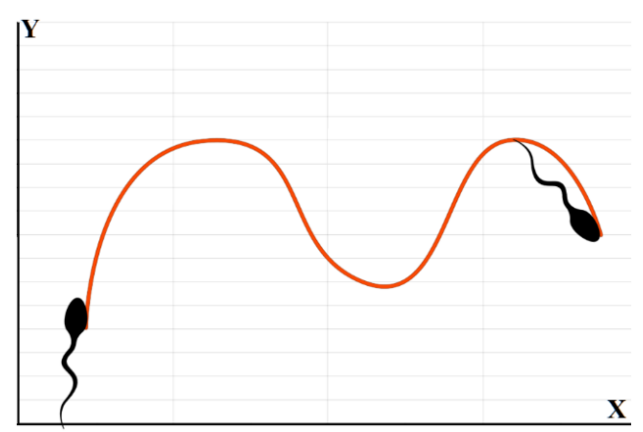

(a)

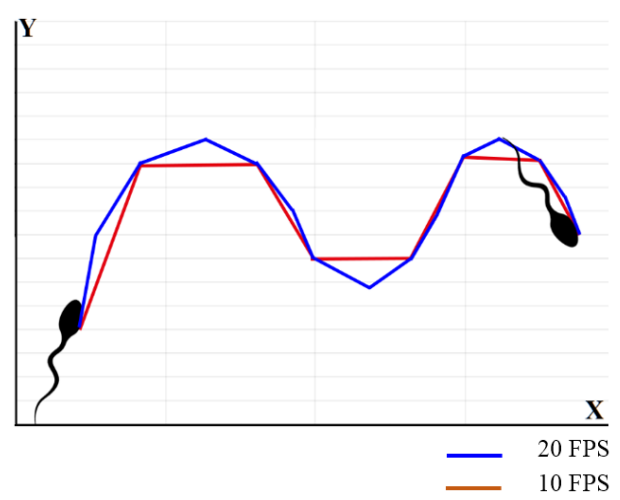

(b)

Figure 2. This figure shows how the video images recorded at different frequencies create different paths for the sperm cells (a) The real-time path of the sperm cell; (b) The path of passing points in different FPS video recordings.

Because the velocity parameters have different results at varied FPS, the CASA systems used currently suggest cameras that record with FPS values appropriate to their system. In their study of Castellini et al. emphasized the necessity of high frame frequency video recordings in order to accurately identify the path of high-speed and non-linear sperm cells (Castellini et al., 2011). They noted that the commercial CASA systems used a frame rate of $30-60 \mathrm{~Hz}$, but this ratio has to be increased to get closer to the real path. Lu et al. proposed in 2013 that the frame rate should still be increased in order to be able to analyze the CASA systems correctly (Lu et al., 2014). In this case, the developed CASA system is dependent on the devices that offer high video frame frequency.

In this study, a polynomial modeling method is presented using Lagrange Interpolation to solve this problem. The known path at the discrete time of sperm can be approximated to its true value by polynomial modeling. In this case, we need to look at polynomial interpolation methods. Scherer's book, published in 2017, suggests that interpolation is necessary when additional data points are needed, for example, to draw a continuous curve (Scherer, 2013).

Lagrange interpolation is a frequently used method to generate continuous polynomial curves. In 2017, Hasan et al. used Lagrange Interpolation to create polynomial models suitable for implementation in the circuit simulator because their derivatives are continuous (Hasan et al., 2017). Sun and Xiong have proposed Lagrange Interpolation for stability analysis of vibrations in hardness variation turning operations (Sun and Xiong, 2017). Lehman et al. have observed that Lagrange Interpolation method in medical imaging is a suitable method (Lehmann et al., 1999).

In this study, VCL and VAP values calculated by classical CASA method and proposed polynomial method in different FPS. The results were compared and presented in Results section. This results showed that it is possible to produce effective results even with low FPS.

In the Material section, information about the preparation process and the hardware structure of the sperm samples are given. In the Methods section, Lagrange Interpolation used in the study and classical calculation methods are presented. In the Polynomial Path Calculation section, the steps of calculating the VAP and VCL parameters using the proposed method are indicated. In the Results section, the results 
of the proposed method and the classical method are compared with Mean Absolute Percentage Error rates.

\section{Materials}

The method of calculating velocity parameters in various species is not different. Of course, the results may vary. However, measuring the distance from one point to another by computer vision requires independent calculation of any species. Therefore, this modeling can also be applied in andrology laboratories. The innovation of this study is to model natural sperm motions in terms of VAP and VCL parameters without being dependent on any species. Thus, the samples used in the study were taken from the Rainbow Trout (Oncorhynchus mykiss) sperm, whose movement behavior was quite complex.

Sperm samples were diluted with an immotile solution (IMS) and activated with motile solution (MS) (Özgür et al., 2018). The dilution ratio was set at 1: 100. All sperm samples were recorded with 20X phase contrast video on the Olympus BX 53, a trinocular microscope. Videos were recorded with CCD Sony VB600B model camera for 5-6 seconds, at least $90 \%$ of the time the cells became immotile. The camera's FPS feature was selected at between 10 and 90 FPS. The concentration of the spermatozoa in the microscope may not be the same in all parts of the sperm sample fluid on the coverslip. However, the sperm can be made homogenous concentration using immotile solution (IMS).

In this context, samples and video recordings were repeated when the homogenous concentration was not stable. With this process, 9 new recordings were created, ranging from 10-90 FPS for each video, using Capture and Video Writer features of OpenCV, an open source image processing library. For sperm analysis, the CASA system developed in 2016 with the project 2140052 supported by TUBITAK-1512 was used (ÖZGÜR et al., 2019). This software gives points of sperm path and uses classical methods presented in the literature as an open source. Since it is not known what kind of methods are used in commercial software, it is not possible to compare with these commercial systems.

The sperm cells in the generated video recordings were tracked using the CASA system. The $(\mathrm{x}, \mathrm{y})$ point information on the path of each sperm cell was obtained with sperm analysis. In Fig. 3, the process of obtaining point-by-point pathways is expressed visually.

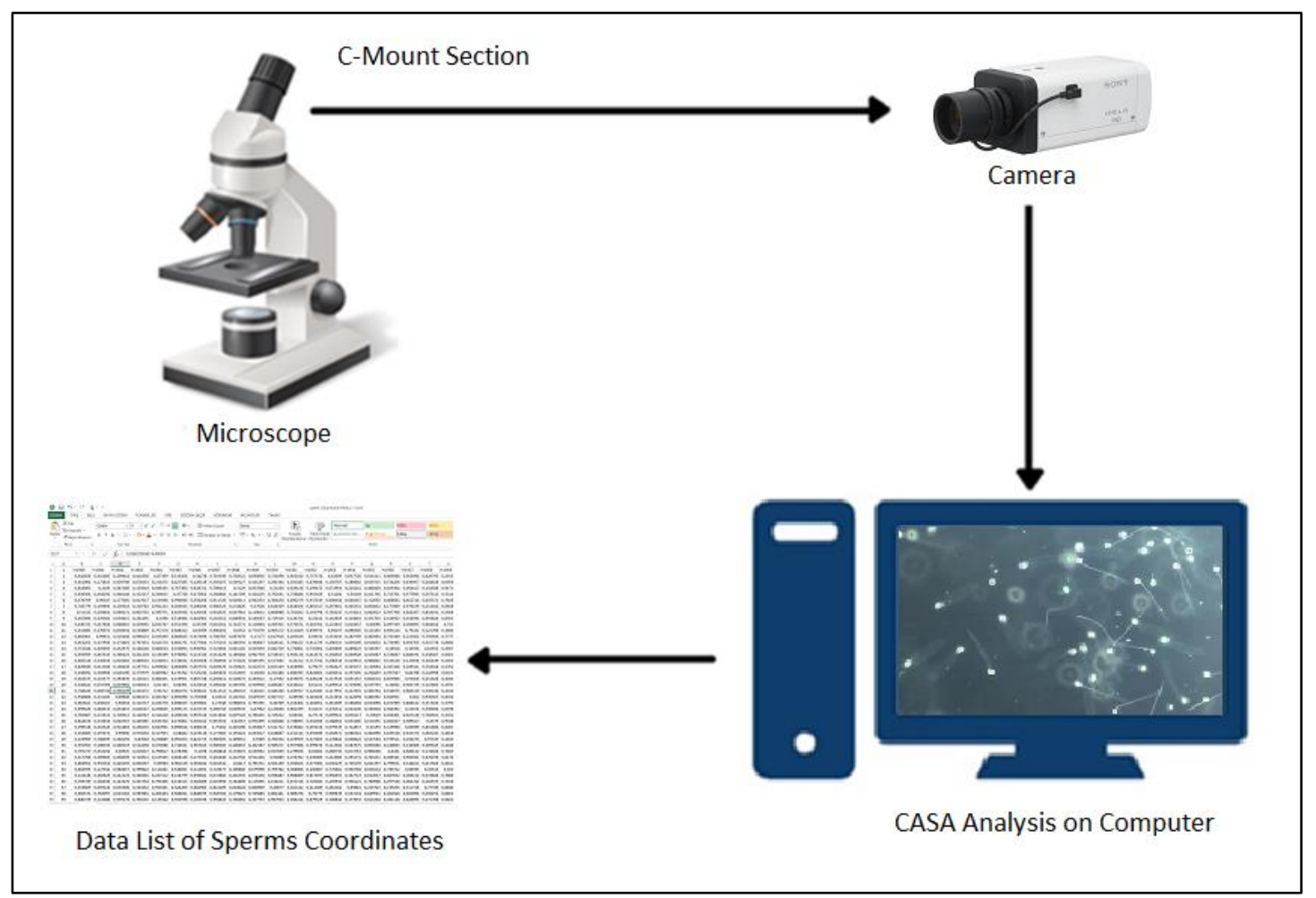

Figure 3. The Process of obtaining sperm path information 


\section{Methods}

\subsection{Lagrange Interpolation}

The Lagrange interpolation can be expressed as a polynomial interpolation and a curve modeling operation. Lagrange interpolations are modeled by a line or curve passing through the point on $x_{i}$ and $y_{i}$. The values of the desired points are calculated on the equation formed by the modeling process. The polynomial grade varies with the number of points. For $n$ points, the polynomial grade is $n-1$. In Fig. 4 , a three-point modeled curve is displayed.

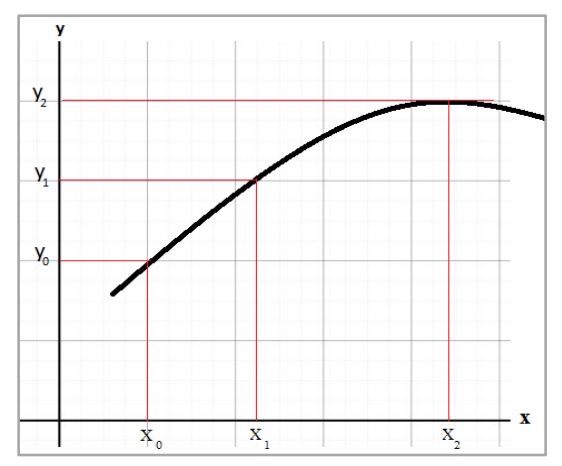

Figure 4. Three-Point Modeled Curve

The definition of Lagrange's polynomial approach is as follows: If a cluster contains $\mathrm{k}+1$ data, points are defined as;

$$
\left(x_{0}, y_{0}\right),\left(x_{1}, y_{1}\right), \ldots,\left(x_{j}, y_{j}\right), \ldots,\left(x_{k}, y_{k}\right)
$$

The Lagrange counterpart of the discrete points is as in Eq. (2.2).

$$
L(x)=\sum_{j=0}^{k} y_{j} l_{j}(x)
$$

In Eq. Hata! Başvuru kaynağı bulunamadı, $l_{j}(x)$ base polynomial is calculated as in Eq. Hata! Başvuru kaynağı bulunamadt.

$$
l_{j}(x)=\prod_{\substack{0 \leq m \leq k \\ m \neq j}} \frac{x-x_{m}}{x_{j}-x_{m}}=\frac{x-x_{0}}{x_{j}-x_{0}} * * * \frac{x-x_{j-1}}{x_{j}-x_{j-1}} \frac{x-x_{j+1}}{x_{j}-x_{j+1}} * * * \frac{x-x_{k}}{x_{j}-x_{k}}
$$

In Eq. Hata! Başvuru kaynağı bulunamadı., since all $x_{j}$ are different from each other, $x_{j}-x_{m} \neq$ 0 at all times, and the equation will work perfectly.

\subsection{Classic VAP and VCL Calculation}

VCL (Curvilinear Velocity) is the curvilinear velocity at which the sperm cell's travels over time. In the VCL calculation, the Euclidean distance relative to the previous position is summed and divided by the total time for each point of sperm path (Hidayatullah et al., 2015; Nieschlag and Behre, 2001). Equation (2.4) shows the VCL calculation.

VAP (Average Path Velocity) is obtained by dividing the path formed by straightening the curvilinear path into time. It is calculated by taking the average of VCL values (Hidayatullah et al., 2015; Nieschlag and Behre, 2001). This parameter gives the knowledge of which gradient the cells go to the target. In equation (2.5), VAP calculation is formulated. 


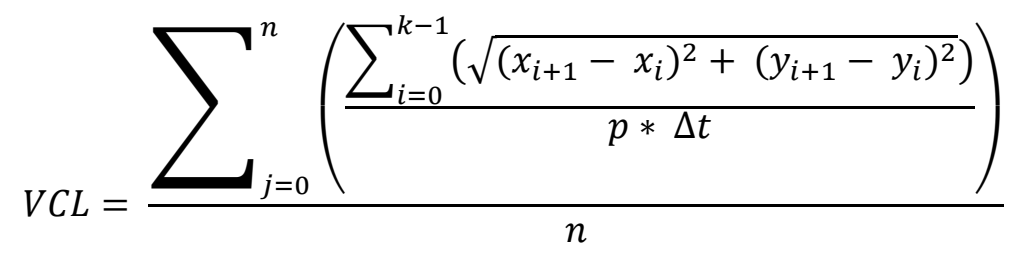

$$
V A P=\frac{\sum_{j=0}^{n}\left(\frac{\sum_{i=0}^{k-1}\left(\sqrt{\left(\overline{x_{l+1}}-\overline{x_{l}}\right)^{2}+\left(\overline{y_{l+1}}-\bar{y}_{l}\right)^{2}}\right)}{p * \Delta t}\right)}{n}
$$

In Equation (2.4) and Equation (2.5), $n$ is the number of sperm, $x$ and $y$ are the points of the path, $k$ is the number of points per path, $p$ is the number of frames, and $\Delta t$ is the time interval between two frames. In equation (2.5), $\bar{x}$ and $\bar{y}$ are the average points of the VCL.

\subsection{Polynomial Path Calculation}

In the literature, VAP and VCL calculations are performed on a discrete-time $(x, y)$ point plane (Hidayatullah et al., 2015). If the sperm cells in motion are moving too fast and the FPS of the camera is low, the distance between the two positions in the image is too great. This positional difference also causes the VAP and VCL values to deviate from their true value. To prevent this situation, a Lagrange based polynomial modeling method was applied in this study.

Although Lagrange Interpolation is an effective method for polynomial curve fitting, there is a problem to be solved in practice. Unbalanced oscillations may occur at extreme points when the degree of polynomial increases. In the literature, this problem is called the Runge Phenomenon. When analyzing sperm video, the number of points per second is quite high compared to the FPS value. When these points are combined with Lagrange, the order of polynomials will be very high. This caused deviations in the endpoints of the curve. In order to avoid this problem, the path points were divided into 10-membered parts respectively. Local solutions were obtained by applying Lagrange Interpolation to the split parts. These local solutions were combined to achieve a general solution. Since the critical issue in the study was the measurement of sperm motions in real time and there is no need an entire polynomial curve, the idea of calculating by dividing it into local parts has produced positive results for both time and error reduction.

Even with low FPS video recording, the result is close to the high FPS performance because this method increases the similarity rate of discrete time path to the continuous time path. This proposed method ensures that we get closer results to reality. The performance achieved by the method applied is shown in Table 1, Fig. 7a and Fig. 7b.

With the CASA system, the location information calculated for each sperm cell is approximated to the real-time path by Lagrange Interpolation, applying the pseudocode shown in Algorithm 1. For an exemplary VCL calculation, the difference, between the paths generated by the classical method and the proposed method, is simulated in Fig. 5. In this simulation, it is seen that the curvilinear line formed at 6 points is different from the line calculated with Euclidean distance. While the curvilinear line in Figure 5 can be obtained by the polynomial approach, CASA systems calculate sperm velocities using Euclidian distance. 


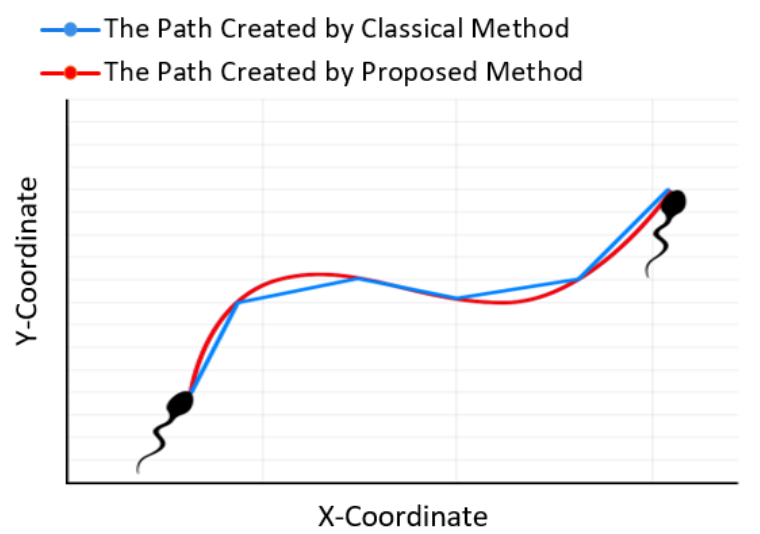

Figure 5. Simulation of 6-points sperm path via proposed and classical method

In the video images analyzed by the CASA system, the sperm cells were plotted as ( $\mathrm{x}, \mathrm{y})$ in each frame and then the curvilinear lengths of the path were obtained by the polynomial approximation algorithm. The pseudocode of the polynomial approximation algorithm for the VAP and VCL velocity parameters are shown in Algorithm 1.

In Algorithm 1, the "CreatePolynomialPath" function is invoked to convert the sperm path to a polynomial form. This function separates the incoming point set into 10-member sub-sets to obtain local Lagrange outputs. By combining these outputs, the global polynomial form is obtained. The used sperm sample mostly involves linear motions and the self-intersection condition is not common when the polynomial is formed. However, considering the sperm of different species and the globalization of the method, the self-intersection problem may occur in the proposed method. The self-intersection of a planar polynomial curve may inadvertently direct the polynomial and lead to errors in the path calculation. In order to solve this problem, a recursive structure to prevent self-intersection was added to the method. In Algorithm1, when the "CheckSelfIntersection" in the function returns true, it means that self-intersection has occurred. To prevent this, the polynomial point set is divided into two equal parts and these two parts are subjected to "GetLocalLagrangian" function recursively. The "GetLocalLagrangian" function calculates the local Lagrange length and returns to the main function. With the recursive feature of this algorithm, control is provided until self-intersection doesn't occurs.

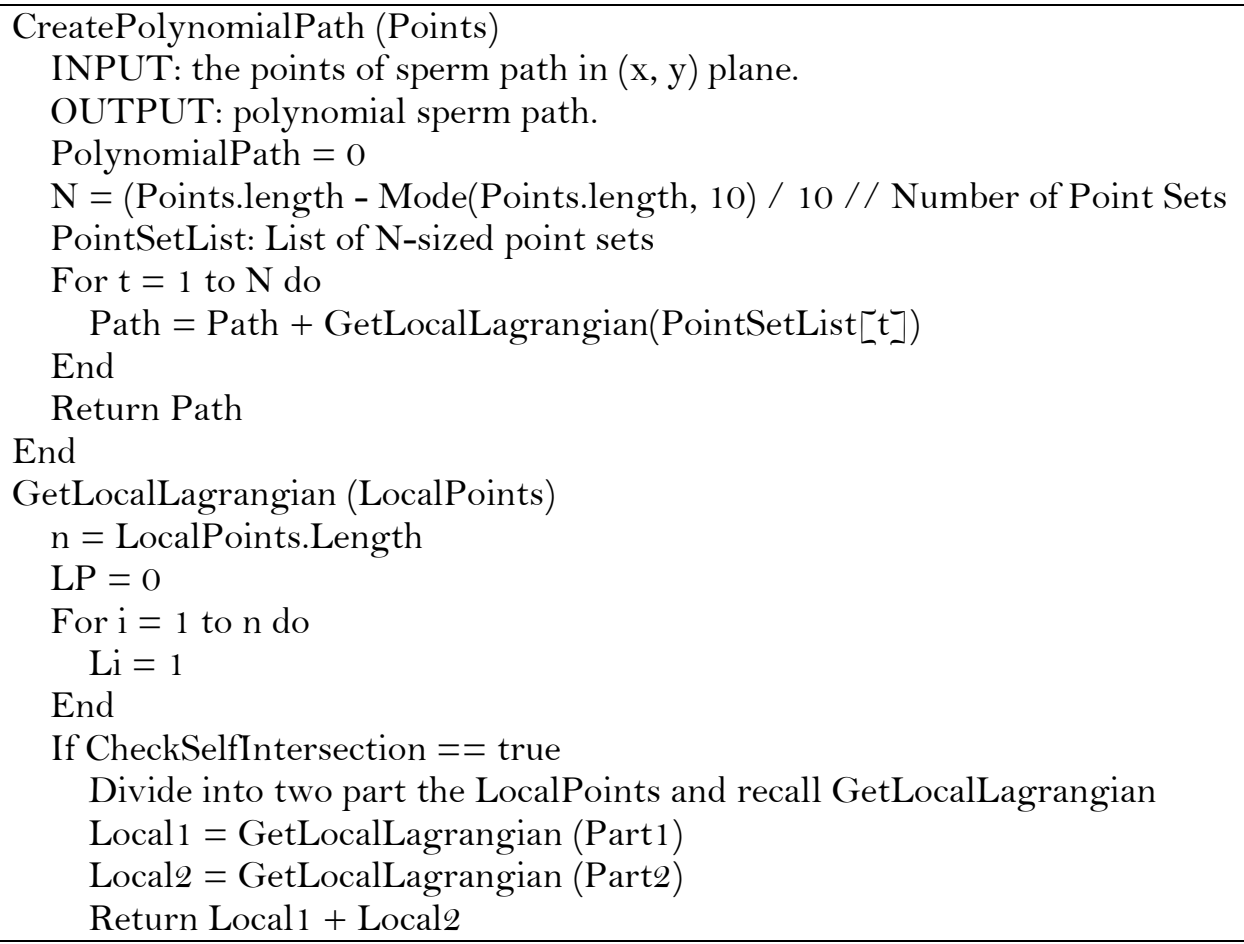




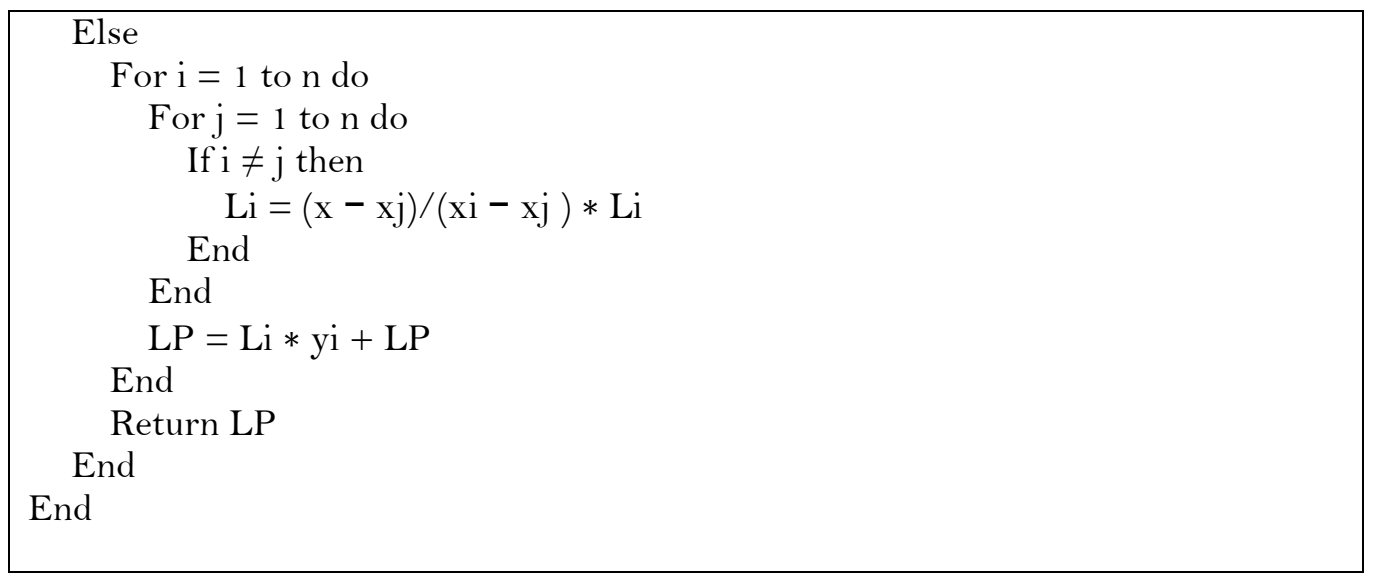

Algorithm 1. Pseudocode of proposed polynomial path calculation method

In Fig. 6, on the real sperm image of 10 FPS, the sperm path of the polynomial calculation method is compared with the classical method. As can be seen here, Fig. 6.a shows the video of 10 FPS, the sperm path calculated by the classical method, and Fig. 6.b shows the calculated sperm path by the polynomial calculation method. The figure shows 10 points length in 1 -second video record via two methods. The velocity of the sample calculated with 10 degrees polynomial in Fig. $6 \mathrm{~b}$. Both images are taken from the same video recording. In 10 frames, 10 position information of the sperm was taken and computed. While the classical method combines the Euclidean distances of the points, the polynomial approach fits a curve by interpolating these points.

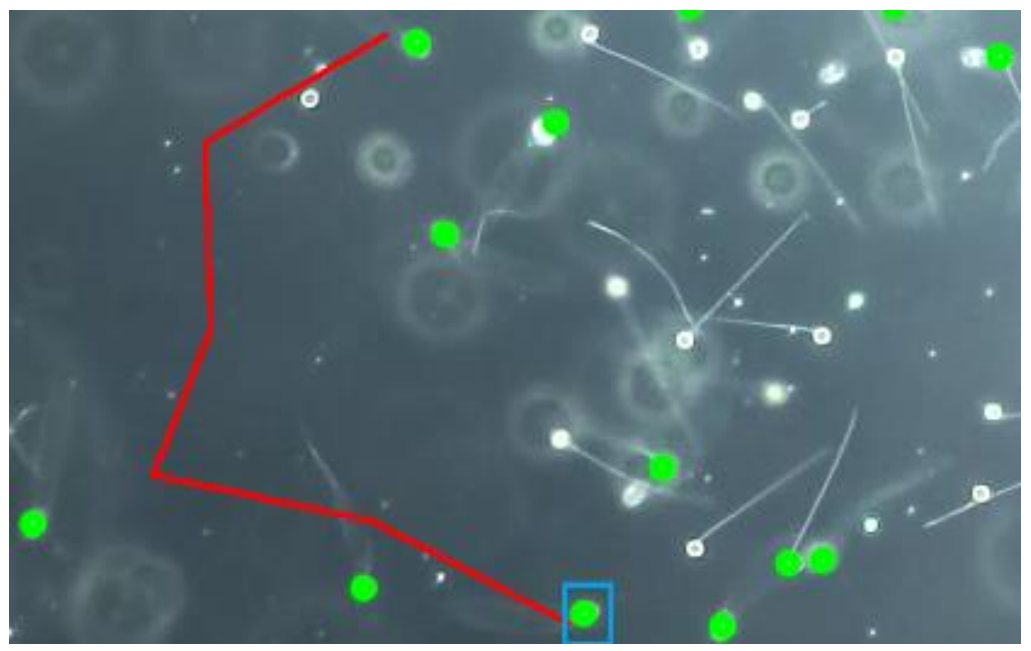

(a)

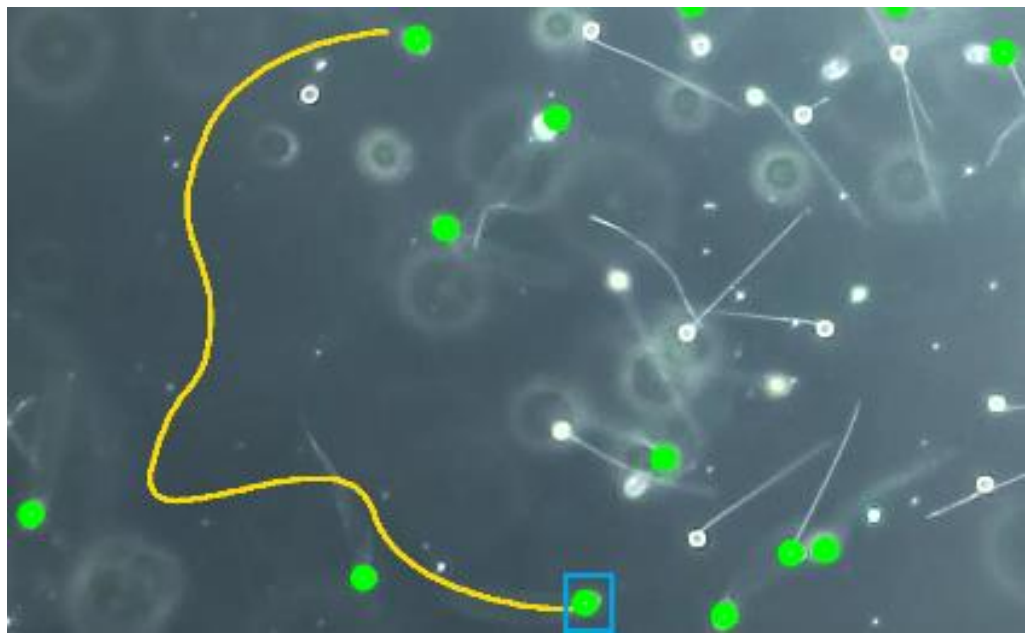


(b)

Figure 6. The path length calculation simulation for 10 FPS video record in 1 second. a) The sperm pathway obtained by the classical method using 10 points. b) The sperm pathway obtained by the polynomial calculation method using 10 points.

\section{Results}

In this study, sperm samples taken from 10 different male rainbow trout had been observed under a microscope and video records were created. Then, video recordings were analyzed by the CASA system. As a result of the analysis, path information, including points of $(x, y)$, was obtained for each sperm cell and a dataset was generated with this information. The dataset was calculated both by the classical method and the proposed method. Calculation for the sperm samples taken from 4 male fish samples is shown in Table 1. As shown in Table 1, point information from video recorded with 9 different FPS for sperm samples taken from 4 different male fish was analyzed. These samples were obtained from laboratory studies by experts in sperm analysis.

A number of tests were performed on 4 different sperm samples to see the effects of the polynomial approach. In these tests, video recordings were obtained at varied frequencies between 10 and 90 frames for each sample sperm. To analyze these samples, the 3-second record selected and was analyzed in the CASA system. The positions information of the sperm was taken as a dataset and sperm velocities were calculated by both methods with this dataset. The results of these tests are shown in Table 1. For the polynomial approach, the point position information of the sperm was divided into 10-items subsets according to the frame order. The starting point of each subset was also divided to be the end point of the previous subset. Local interpolations were calculated for the subsets and general interpolation was created by the addition of these local interpolations. As the first line in Table 1 was taken as an example, 3 -second video was recorded on 10 FPS for Sperm-1. This 10 FPS video has 30 frames for 3 seconds and 30 transition points. There are three 10-items point sets and a set of 4 -items point set at these 30 transition points. Therefore, 4 polynomial computations were performed for 10 FPS and 3 seconds of video recording. The effect of the polynomial approach in the first row of Table 1 is $38.89 \%$ closer to the real length compared to the conventional method.

The results show that the mean absolute error of the lowest frequency (10 FPS) is $18.32 \%$ in the proposed method, whereas it is $49.88 \%$ in the classical method. For the 20 FPS video records, the average absolute error of the proposed polynomial method was $12.02 \%$ while it was found to be $36.60 \%$ by the classical method. This information suggests that the polynomial method produces more effective results with low FPS video records.

In Fig. 7, the effect of the proposed method is shown. In the experimental results, the VCL value obtained at frequencies of 90 FPS and above is fixed in both methods. From here, it is assumed that the value obtained at 90 FPS is the threshold for the real-time VCL value. The threshold point can also change when the test environment changes. The error percentages in Table 1, are calculated according to this threshold. In Fig. 7, the proposed method was calculated by taking the arithmetic mean of the extrapolation results for each sample. The classical method was calculated by taking the arithmetic mean of the classical method results for each sample.

VCL or VAP values for real-time can't be obtained precisely. What CASA systems do is to produce results that are close to real-time velocity. However, in the classical CASA systems, the way to get closer to the real-time VCL is to increase the frame frequency of the video. As can be seen in Fig. 7a, the results of the polynomial approach are closer to the threshold value even at low FPS. Mean Absolute Percent Errors (MAPE) for both methods are shown in Fig. 7b. Although the polynomial approach produces positive results for real-time velocity calculation, it requires a high cost of calculation as seen in Table 1. 


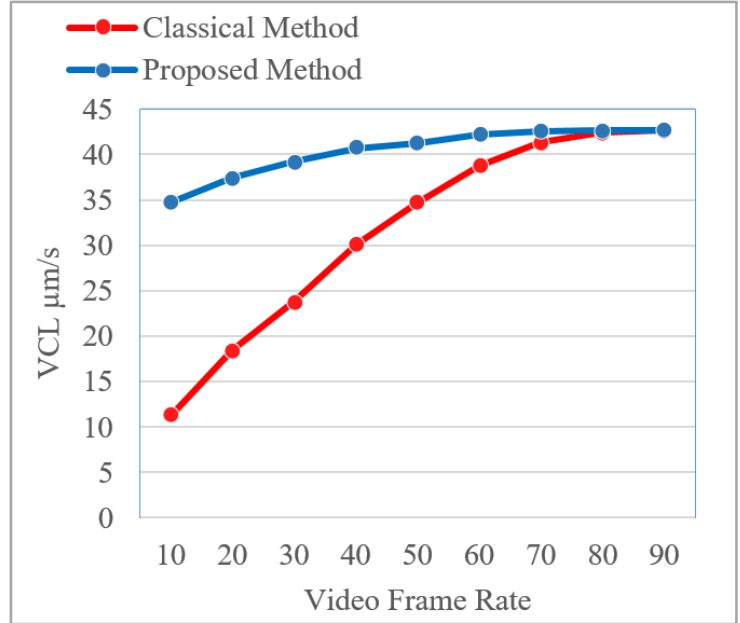

(a)

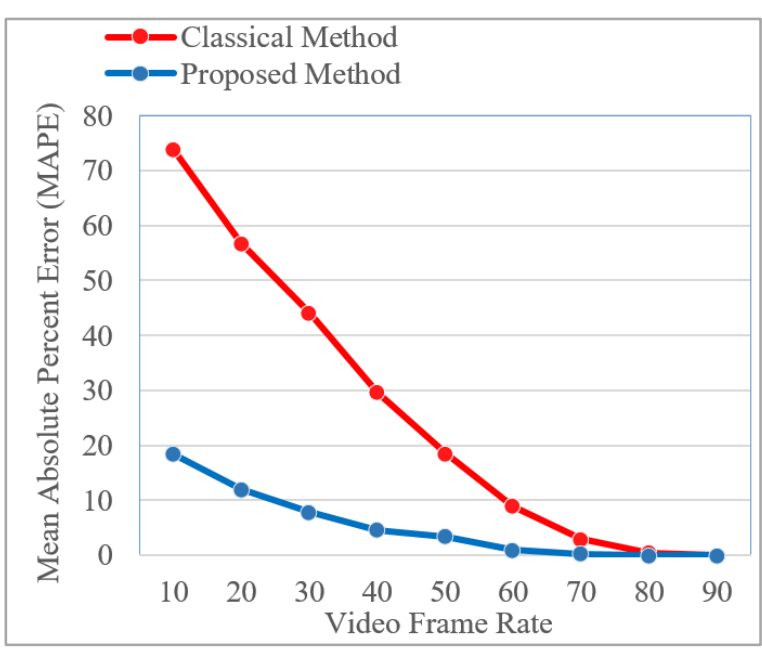

(b)

Figure 7. (a) Comparison of mean VCL values with classical and proposed methods in different FPSs; (b) MAPE Curves for Both Methods in Different Frame Ratios. The proposed method results are obtained from the average of Extrapolation results.

Table 1. Comparison of the Proposed Method and the Classical Method for 4 Male Fish using 3second videos.

\begin{tabular}{|c|c|c|c|c|c|c|c|c|}
\hline FPS & $\begin{array}{c}\text { Number } \\
\text { of } \\
\text { Points }\end{array}$ & $\begin{array}{c}\text { Num. of } \\
\text { Polynomial } \\
(10 \\
\text { degrees }) \\
\end{array}$ & $\begin{array}{c}\text { Classical } \\
\text { Method } \\
(\mathrm{VCL} \\
\mu \mathrm{m} / \mathrm{s})\end{array}$ & $\begin{array}{c}\text { C.M. } \\
\text { Execution } \\
\text { Time } \\
\text { (sec.) }\end{array}$ & $\begin{array}{c}\text { C.M. } \\
\text { Absolute } \\
\text { Error } \\
(\%) \\
\end{array}$ & $\begin{array}{c}\text { Proposed } \\
\text { Method } \\
(\mathrm{VCL} \\
\mu \mathrm{m} / \mathrm{s}) \\
\end{array}$ & $\begin{array}{c}\text { P.M. } \\
\text { Execution } \\
\text { Time } \\
\text { (sec.) }\end{array}$ & $\begin{array}{c}\text { P.M. } \\
\text { Absolute } \\
\text { Error } \\
(\%)\end{array}$ \\
\hline \multicolumn{9}{|c|}{ Sperm Number: 1} \\
\hline 10 & 30 & 4 & 19,8489 & 0.0529 & 52.9944 & 36.2748 & 0.2048 & 14.0951 \\
\hline 20 & 60 & 7 & 24,9151 & 0.1001 & 40.9968 & 37.3174 & 0.5011 & 11.6261 \\
\hline 30 & 90 & 11 & 27,4323 & 0.1495 & 35.0357 & 38.3322 & 0.9489 & 9.2228 \\
\hline 40 & 120 & 15 & 29,0722 & 0.2078 & 31.1521 & 39.3415 & 1.5060 & 6.8326 \\
\hline 50 & 150 & 18 & 31,3292 & 0.2388 & 25.8071 & 40.3496 & 2.3458 & 4.4453 \\
\hline 60 & 180 & 21 & 36,4563 & 0.2789 & 13.6653 & 41.3420 & 3.2807 & 2.0951 \\
\hline 70 & 210 & 25 & 39,9732 & 0.3089 & 5.3367 & 42.0826 & 4.5218 & 0.3413 \\
\hline 80 & 240 & 28 & 41,6507 & 0.3317 & 1.3641 & 42.1993 & 6.1538 & 0.0649 \\
\hline 90 & 270 & 31 & 42,2132 & 0.3573 & 0.0320 & 42.2267 & 8.2645 & 0 \\
\hline \multicolumn{9}{|c|}{ Sperm Number: 2} \\
\hline 10 & 30 & 4 & 18,9717 & 0.0499 & 49.6260 & 33.6518 & 0.1985 & 10.6472 \\
\hline 20 & 60 & 7 & 25,7994 & 0.0901 & 31.4970 & 35.1935 & 0.4507 & 6.5536 \\
\hline 30 & 90 & 11 & 28,5968 & 0.1398 & 24.0693 & 36.1015 & 0.9301 & 4.1427 \\
\hline 40 & 120 & 15 & 30,5965 & 0.2054 & 18.7596 & 36.8224 & 1.5746 & 2.2285 \\
\hline 50 & 150 & 18 & 33,1313 & 0.2395 & 12.0292 & 37.0355 & 2.4044 & 1.6627 \\
\hline 60 & 180 & 21 & 35,5491 & 0.2892 & 5.6094 & 37.5002 & 3.4082 & 0.4288 \\
\hline 70 & 210 & 25 & 36,8879 & 0.3055 & 2,0546 & 37.6254 & 4.7343 & 0.0964 \\
\hline 80 & 240 & 28 & 37,5211 & 0.3328 & 0,3733 & 37.6593 & 6.5047 & 0.0064 \\
\hline 90 & 270 & 31 & 37,6588 & 0.3758 & 0,0077 & 37.6617 & 9.0039 & 0 \\
\hline \multicolumn{9}{|c|}{ Sperm Number: 3} \\
\hline 10 & 30 & 4 & 21,6200 & 0.0565 & 49,8441 & 34.0013 & 0.1894 & 21.1209 \\
\hline 20 & 60 & 7 & 26,4875 & 0.1012 & 38,5521 & 36.2564 & 0.4613 & 15.8894 \\
\hline 30 & 90 & 11 & 30,0560 & 0.1465 & 30,2736 & 38.1028 & 0.9287 & 11.6059 \\
\hline 40 & 120 & 15 & 33,9842 & 0.2093 & 21,1606 & 40.2840 & 1.6143 & 6.5458 \\
\hline 50 & 150 & 18 & 36,1840 & 0.2651 & 16,0573 & 40.3496 & 2.5817 & 6.3936 \\
\hline 60 & 180 & 21 & 38,3225 & 0.2990 & 11,0962 & 42.9584 & 3.7002 & 0.3415 \\
\hline 70 & 210 & 25 & 41,9980 & 0.3265 & 2,5695 & 43.0544 & 5.1159 & 0.1188 \\
\hline 80 & 240 & 28 & 43,0750 & 0.3468 & 0,0710 & 43.1025 & 6.8661 & 0.0072 \\
\hline
\end{tabular}




\begin{tabular}{ccccccccc}
\hline 90 & 270 & 31 & 43,1006 & 0.3508 & 0,0116 & 43.1056 & 9.5282 & 0 \\
\hline \multicolumn{7}{c}{ Sperm Number: 4} \\
10 & 30 & 4 & 23,9846 & 0.0422 & 48,5318 & 37.0023 & 0.1433 & 20.5973 \\
20 & 60 & 7 & 28,6651 & 0.0855 & 38,4880 & 41.1088 & 0.3851 & 11.7852 \\
30 & 90 & 11 & 32,0254 & 0.1240 & 31,2771 & 43.4458 & 0.8928 & 6.7703 \\
40 & 120 & 15 & 35,2180 & 0.1749 & 24,4262 & 44.9760 & 1.4362 & 3.4866 \\
50 & 150 & 18 & 37,8742 & 0.2383 & 18,7263 & 45.3475 & 2.2246 & 2.6894 \\
60 & 180 & 21 & 41,0049 & 0.2791 & 12,0082 & 45.9002 & 3.4662 & 1.5034 \\
70 & 210 & 25 & 44,9844 & 0.3078 & 3,4686 & 46.2991 & 4.9888 & 0.6474 \\
80 & 240 & 28 & 46,4580 & 0.3312 & 0,3064 & 46.5986 & 6.2009 & 0.0047 \\
90 & 270 & 31 & 46,5982 & 0.3497 & 0,0056 & 46.6008 & 8.7003 & 0 \\
\hline \hline
\end{tabular}

In Table 1;

FPS: For each sperm sample, the video records have the number of frames per second.

Number of Points: The number of points that the sperm passed through for 3 seconds.

Number of Polynomial: Subsets and number of polynomials applied to these subsets.

Classical Method $(\mu \mathrm{m} / \mathrm{s})$ : The VCL result performed by the classical method with the specified FPS.

C.M. Execution Time (sec.): The calculation time with the classical method.

C.M. Absolute Error (\%): The absolute error with the classical method.

Proposed Method $(\mu \mathrm{m} / \mathrm{s})$ : The result performed by the proposed polynomial method with the specified FPS.

P.M. Execution Time (sec.): The calculation time with the proposed method.

P.M. Absolute Error (\%): The absolute error with the proposed method.

VCL or VAP values for real-time can't be obtained precisely. What CASA systems do is to produce results that are close to real-time velocity. However, in the classical CASA systems, the way to get closer to the real-time VCL is to increase the frame frequency of the video. As can be seen in Fig. 7a, the results of the polynomial approach are closer to the threshold value even at low FPS. Mean Absolute Percent Errors (MAPE) for both methods are shown in Fig. 7b. Although the polynomial approach produces positive results for real-time velocity calculation, it requires a high cost of calculation as seen in Table 1.

\section{Discussions}

In this study, the discrete calculation model of the classical method was considered as a problem in the calculation of sperm velocity. Thus, polynomial modeling was applied to produce a solution to this problem. This study focuses on the VCL and VAP parameters of the sperm velocity analysis. The samples used in the study were taken from trout sperm which had a very complicated movement behavior. Since the calculation formula for sperm velocity parameters is the same for all species that reproduce by sexual reproduction, the proposed polynomial modeling method in the study can be applied to other species. This study deals with the development of general velocity calculation methods, not the behavior of a specific species.

Lagrange Interpolation was used for polynomial modeling. In order to eliminate the oscillation problem at the endpoints encountered in the high-order polynomial calculations of Lagrange Interpolation, the points were divided into the 10-membered local parts. Lagrange polynomial can be calculated more effectively for minimized points. These local solutions are combined and a general solution was obtained. It can be thought that calculating all interpolation with local solutions will bring an insensitive result, but the method even produces effective results compared to the classical method.

In order to solve the problem of self-intersection, the method was checked recursively. In this way, corrections were made in polynomial until this problem did not occur. Although the proposed method has a high performance for real path length, the calculation cost is high compared to the classical method. This situation poses a disadvantage in terms of calculation costs for this study.

As a result of the study, it was found that the polynomial modeling method for the calculation of velocity parameters showed higher performance than the classical method, especially with low FPS. Wilson-Leedy and Ingermann noted that the VCL parameters generated by varied FPS records are 
different from each other. This study shows that the polynomial approach can produce similar results in varied FPS.

In this study, the mean absolute error of the results was $18.32 \%$ with polynomial modeling for 10 FPS recordings. At the same frame frequency, the mean absolute error of the results was $49.88 \%$ by the classical method. Since the number of points forming the sperm path increases at higher frequencies, the difference between the two methods decreases when FPS increases. The percentage of absolute error is calculated by considering the values at a very high frequency as the threshold point. The actual length of the sperm path is not known because of the discrete time processing of the computer vision, thus, a threshold value for the actual path length is applied in this study.

The classical methods used in the study were taken from the studies presented in the literature as an open source. Since it is not known what kind of methods are used in commercial software, it is not possible to compare with these systems.

Sperm analysis methods in the literature are calculated by basing the Euclidean distance of the points. This leads to a certain amount of compromise from the actual road account. Because, in our observations, it was observed that the sperms followed a path with a polynomial curve rather than a linear line. Therefore, it is predicted that polynomial approaches will produce more realistic results than classical methods for measuring sperm pathways. Furthermore, it is necessary to use high FPS cameras in order to produce realistic results by classical method. In this case, the need arises for devices with high processing capacity. This is a disadvantage of the classical method applied in CASA systems.

The results obtained in the study have shown that even with low FPSs, realistic results are obtained with the proposed method. As a result of the study; in order to obtain more realistic results, it is recommended to use polynomial approaches in sperm velocity calculations in CASA systems. With this study, CASA systems will be able to get rid of the hardware dependency caused by the camera and provide the correct information by creating a natural movement model. In future studies, a simulation software, in which virtual sperm cells are activated, will be performed to better test the method. Thus, since the actual path will be known with this simulation software, there will be no need to apply a threshold value. To reduce the cost of calculation, which is enhanced by polynomial modeling, is also among our targets.

\section{References}

Ahn, H., Cho, H.-J., 2019. Research of multi-object detection and tracking using machine learning based on knowledge for video surveillance system. Pers. Ubiquitous Comput. https://doi.org/10.1007/s00779-019-01296-z

Alquézar-Baeta, C., Gimeno-Martos, S., Miguel-Jiménez, S., Santolaria, P., Yániz, J., Palacín, I., Casao, A., Cebrián-Pérez, J.Á., Muiño-Blanco, T., Pérez-Pé, R., 2019. OpenCASA: A new open-source and scalable tool for sperm quality analysis. PLOS Comput. Biol. 15, e1006691. https://doi.org/10.1371/journal.pcbi.1006691

Amann, R.P., Waberski, D., 2014. Computer-assisted sperm analysis (CASA): Capabilities and potential developments. Theriogenology 51, https://doi.org/10.1016/j.theriogenology.2013.09.004

Boe-Hansen, G.B., Satake, N., 2019. An update on boar semen assessments by flow cytometry and CASA. Theriogenology. https://doi.org/10.1016/j.theriogenology.2019.05.043

Bompart, D., García-Molina, A., Valverde, A., Caldeira, C., Yániz, J., Núñez de Murga, M., Soler, C., 2018. CASA-Mot technology: how results are affected by the frame rate and counting chamber. Reprod. Fertil. Dev. 30, 810. https://doi.org/10.1071/RD17551

Bouwmans, T., 2014. Traditional and recent approaches in background modeling for foreground detection: An overview. Comput. Sci. Rev. https://doi.org/10.1016/j.cosrev.2014.04.001

Castellini, C., Dal Bosco, A., Ruggeri, S., Collodel, G., 2011. What is the best frame rate for evaluation of sperm motility in different species by computer-assisted sperm analysis? Fertil. Steril. 96, 24 


\section{7. https://doi.org/10.1016/J.FERTNSTERT.2011.04.096}

Cocorullo, G., Corsonello, P., Frustaci, F., Guachi-Guachi, L. de los A., Perri, S., 2016. Multimodal background subtraction for high-performance embedded systems. J. Real-Time Image Process. 117. https://doi.org/10.1007/s11554-016-0651-6

Contri, A., Valorz, C., Faustini, M., Wegher, L., Carluccio, A., 2010. Effect of semen preparation on casa motility results in cryopreserved bull spermatozoa. Theriogenology 74, 424-435. https://doi.org/10.1016/j.theriogenology.2010.02.025

Duffy, B., Thiyagalingam, J., Walton, S., Smith, D.J., Trefethen, A., Kirkman-Brown, J.C., Gaffney, E.A., Chen, M., 2015. Glyph-Based Video Visualization for Semen Analysis. IEEE Trans. Vis. Comput. Graph. 21, 980-993. https://doi.org/10.1109/TVCG.2013.265

Elgammal, A., Duraiswami, R., Harwood, D., Davis, L.S., 2002. Background and foreground modeling using nonparametric kernel density estimation for visual surveillance. Proc. IEEE 90, 1151-1162. https://doi.org/10.1109/JPROC.2002.801448

Hasan, M.S., Rahman, T., Islam, S.K., Blalock, B.B., 2017. Numerical modeling and implementation in circuit simulator of SOI four-gate transistor (G4FET) using multidimensional Lagrange and $\begin{array}{lllll}\text { Bernstein } & \text { polynomial. } & \text { Microelectronics } & \text { J. } & 65,\end{array}$ https://doi.org/10.1016/J.MEJO.2017.05.011

Hidayatullah, P., Awaludin, I., Kusumo, R.D., Nuriyadi, M., 2015. Automatic sperm motility measurement, in: 2015 International Conference on Information Technology Systems and Innovation (ICITSI). IEEE, pp. 1-5. https://doi.org/10.1109/ICITSI.2015.7437674

Hu, F., Fan, J., Luo, K., Zhou, Y., Wu, C., Luo, L., Wang, S., Tao, M., Zhang, C., Chen, B., Ma, M., Liu, S., 2019. Comparative analyses of reproductive characteristics of functional sex reversal male gynogenetic red crucian carp and ordinary male red crucian carp. Aquaculture 511. https://doi.org/10.1016/j.aquaculture.2019.06.013

Khalifa, N.E.M., Taha, M.H.N., Hassanien, A.E., 2019. Automatic Counting and Visual Multi-tracking System for Human Sperm in Microscopic Video Frames. Springer, Cham, pp. 525-531. https://doi.org/10.1007/978-3-319-99010-1_48

Kř́žzová, J., Čoudková, V., Maršálek, M., 2017. Computer-Assisted Sperm Analysis of Head Morphometry and Kinematic Parameters in Warmblood Stallions Spermatozoa. J. Equine Vet. Sci. 57, 8-17. https://doi.org/10.1016/J.JEVS.2017.05.012

Lehmann, T.M., Gonner, C., Spitzer, K., 1999. Survey: interpolation methods in medical image processing. IEEE Trans. Med. Imaging 18, 1049-1075. https://doi.org/10.1109/42.816070

Lu, J.C., Huang, Y.F., Lü, N.Q., 2014. Computer-aided sperm analysis: past, present and future. Andrologia 46, 329-338. https://doi.org/10.1111/and.12093

Nieschlag, E., Behre, H.M., 2001. Andrology : Male Reproductive Health and Dysfunction. Springer Berlin Heidelberg.

Özgür, M.E., Balcığlu, S., Ulu, A., Özcan, İ., Okumuş, F., Köytepe, S., Ateş, B., 2018. The in vitro toxicity analysis of titanium dioxide ( $\mathrm{TiO} 2$ ) nanoparticles on kinematics and biochemical quality of rainbow trout sperm cells. Environ. Toxicol. Pharmacol. https://doi.org/10.1016/j.etap.2018.06.002

ÖZGÜR, M.E., OKUMUŞ, F., KOCAMAZ, A.F., 2019. A Novel Computer Assisted Sperm Analyzer for Assessment of Spermatozoa Motility in Fish; BASA-Sperm Aqua. El-Cezeri Fen ve Mühendislik Derg. 6, 208-219. https://doi.org/10.31202/ecjse.486342

Qi, S., Nie, T., Li, Q., He, Z., Xu, D., Chen, Q., 2019. A Sperm Cell Tracking Recognition and Classification Method. Institute of Electrical and Electronics Engineers (IEEE), pp. 163-167. 
https://doi.org/10.1109/iwssip.2019.8787312

Rurangwa, E., Kime, D.E., Ollevier, F., Nash, J.P., 2004. The measurement of sperm motility and factors affecting sperm quality in cultured fish. Aquaculture. https://doi.org/10.1016/j.aquaculture.2003.12.006

Scherer, P.O.J., 2013. Interpolation. pp. 15-35. https://doi.org/10.1007/978-3-319-00401-3_2

Sobral, A., Vacavant, A., 2014. A comprehensive review of background subtraction algorithms evaluated with synthetic and real videos. Comput. Vis. Image Underst. 122, 4-21. https://doi.org/10.1016/j.cviu.2013.12.005

Stauffer, C., Grimson, W.E.L., 1999. Adaptive background mixture models for real-time tracking. Proc. IEEE Comput. Soc. Conf. Comput. Vis. Pattern Recognit. 2, 246-252. https://doi.org/10.1109/cvpr.1999.784637

Sun, Y., Xiong, Z., 2017. High-order full-discretization method using Lagrange interpolation for stability analysis of turning processes with stiffness variation. J. Sound Vib. 386, 50-64. https://doi.org/10.1016/J.JSV.2016.08.039

Urbano, L.F., Masson, P., VerMilyea, M., Kam, M., 2017. Automatic Tracking and Motility Analysis of Human Sperm in Time-Lapse Images. IEEE Trans. Med. Imaging 36, 792-801. https://doi.org/10.1109/TMI.2016.2630720

Valverde, A., Madrigal, M., Caldeira, C., Bompart, D., de Murga, J.N., Arnau, S., Soler, C., 2019. Effect of frame rate capture frequency on sperm kinematic parameters and subpopulation structure definition in boars, analysed with a CASA-Mot system. Reprod. Domest. Anim. 54, 167-175. https://doi.org/10.1111/rda.13320

Wilson-Leedy, J.G., Ingermann, R.L., 2007. Development of a novel CASA system based on open source software for characterization of zebrafish sperm motility parameters. Theriogenology 67, 661-672. https://doi.org/10.1016/J.THERIOGENOLOGY.2006.10.003

World Health Organization, 2010. WHO laboratory manual for the Examination and processing of human semen, World Health Organization. https://doi.org/10.1038/aja.2008.57

Yamasaki, K., Watanabe, N., Ihana, T., Ishijima, S., Fujiwara, T., Tsutsumi, O., Iwamoto, T., 2017. MP07-10 USEFULNESS OF A PORTABLE COMPUTER-ASSISTED SPERM ANALYZER SYSTEM USING SMARTPHONE. J. Urol. 197. https://doi.org/10.1016/j.juro.2017.02.276

Yániz, J.L., Palacín, I., Vicente-Fiel, S., Sánchez-Nadal, J.A., Santolaria, P., 2015. Sperm population structure in high and low field fertility rams. Anim. Reprod. Sci. 156, 128-134. https://doi.org/10.1016/J.ANIREPROSCI.2015.03.012 